\title{
Caracterización de la dinámica de las relaciones de género en la Escuela Naval de Cadetes "Almirante Padilla"
}

\author{
Martha Catalina Gualdrón Castillo, Eduardo Ahoron Waite Samudio \& Cedrid Gómez Torregrosa \\ Escuela Naval de Cadetes "Almirante Padilla”, Cartagena, Colombia. \\ mcatalina109@gmail.com, eduardowaite1027@hotmail.com, cedrid.gomez@armada.mil.co
}

Recibido: Junio 16, 2020.

Recibido en su versión corregida: Agosto 12, 2020.

Aceptación: Septiembre 09, 2020.

Cómo citar: Gualdrón Castillo, M.C., Waite Samudio, E.A. \& Gómez Torregrosa, C. (2020). Caracterización de la dinámica de las relaciones de género en la Escuela Naval de Cadetes “Almirante Padilla”. Revista Sextante, 23, pp. 28 - 36, 2020.

\section{Resumen}

Para saber cómo es la dinámica de las relaciones de género con el ingreso de la mujer a la Escuela Naval de Cadetes "Almirante Padilla", se caracterizó la misma por medio de una investigación descriptiva con método inductivo y enfoque cualitativo etnográfico, específicamente escolar militar; utilizando entrevista y encuestas. Se hace necesario tener en cuenta cómo es la relación entre hombres y mujeres en el desarrollo de cualquier actividad. El ingreso de la mujer se reflejó como una oportunidad de desarrollo profesional en los diferentes ámbitos que desempeña. Esto requirió una inversión, pues debió ser modificada gran parte de su estructura, además el cambió de pensamiento fue algo que costó mucho trabajo, ya que no fue fácil modificar los ideales de una cultura de un día para otro, pero igual es algo que debió suceder ya que todo debe ir cambiando y mejorando.

Palabras claves: Dinámica, Género, Inclusión Militar, Mujer Naval.

\section{Dynamics characterization of gender relations at the Escuela Naval de Cadetes "Almirante Padilla"}

\begin{abstract}
To find out how the dynamics of gender relations are with the entrance of women to the Naval Cadet School "Almirante Padilla", it was characterized through descriptive research with an inductive method and a qualitative ethnographic approach, specifically military school; using interview and surveys. It is necessary to take into account the relationship between men and women in the development of any activity. The income of women was reflected as an opportunity for professional development in the different areas that it carries out, although it is true that this required an investment, since a large part of its structure had to be modified, also the change of thought was something that cost a lot work, since it was not easy to modify the ideals of a culture from one day to the next, but it is still something that should have happened since everything must be changing and improving.
\end{abstract}

Keywords: Dynamics, Gender, Inclusion, Military, Naval Woman. 


\section{Introducción}

Preguntarse ¿Cómo es la dinámica de las relaciones de género con el ingreso de la mujer a la Escuela Naval de Cadetes "Almirante Padilla" ?, es el motivo que trata de abordar este proyecto de investigación, debido a la poca importancia que se había dado a las relaciones de género en la misión que tiene la mujer en la Escuela Naval.

Este trabajo se realizó con el fin de enmarcar cómo han evolucionado las relaciones de género en la Escuela Naval de Cadetes Almirante Padilla desde la admisión de la mujer, tanto administrativa, como de línea; estableciendo la evolución del trato que se les ha dado a las mismas, como consecuencia de la igualdad de género, teniendo en cuenta que un hombre no tiene las mismas características de una mujer, y la mujer puede desarrollar muchas habilidades en poco tiempo de acuerdo a las necesidades del entorno. La importancia de la investigación es enmarcar los conocimientos culturales navales en cuanto a la evolución de las relaciones de género. Es de gran importancia debido a que no existen trabajos relacionados que describan la relación de género en el ámbito naval militar.

\section{Metodología}

Escuela Naval de Cadetes "Almirante Padilla". Luego se aplicaron entrevistas y encuestas abiertas a a una muestra de oficiales y alumnos del batallón, con fin de identificar cómo un género visualiza el papel del otro y cómo es el trato entre ambos. Además, se utilizó el apoyo bibliográfico y se analizaron las herramientas con las que cuenta la Escuela Naval de Cadetes "Almirante Padilla" para la inclusión de género.

Para el análisis de los resultados de la encuesta se aplicó la fórmula cálculo de la muestra para poblaciones finitas arrojando un resultado de 150 cadetes, siendo la población objeto de estudio 572, la cual es el total actual de alumnos del batallón, sin contar la compañía de los aspirantes quienes por su antigüedad aún no comparten con el resto de los cadetes y no se relacionan con los mismos.

\section{Resultados}

A lo largo de la historia la mujer se ha ido incorporando a las instituciones militares en situaciones bélicas, en las que se hacía especialmente necesaria el aporte de todos los recursos humanos disponibles en orden a posibilitar la defensa nacional. El primer país que integró de modo estable a la mujer en las Fuerzas Armadas fue el Reino Unido con la creación, en 1881, del Servicio Imperial de Enfermeras, ejemplo seguido ocho años después por Canadá. En el año 1917 se procedía a incorporar a la mujer a otros ámbitos de la organización castrense, como transmisiones y Estado Mayor. La Segunda Gran Guerra no podía, por menos, que incidir sobre esta situación. En 1940 con Francia ocupada por los ejércitos del Reich, se creaba el Servicio Nacional de Auxiliares Femeninas, transformado en 1941 en el Cuerpo de Voluntarias Francesas. En 1942, en Noruega, se adoptaba una decisión histórica, como bien se ha puesto de relieve, al decretar el servicio militar obligatorio para las mujeres. Ello era tributario, como es obvio, de la situación de emergencia que provenía de la ocupación alemana, manteniéndose esta norma tan sólo hasta 1945.

La presencia de las mujeres cadetes en la Armada Colombiana se hizo efectiva a partir del 13 de enero de 1984, siguiendo los pasos de otros países suramericanos como Chile, Argentina y Venezuela. Esta dejó de ser una actividad exclusiva de hombres, cuando por primera vez, hace ya más de un siglo, ingresaran las primeras mujeres a la Armada; sin embargo, aún persiste el que sean ellos quienes ocupen los cargos más altos, y los datos lo demuestran. (FF SALGADO, 2000)

Las primeras cadetes de la Armada se graduaron como administradoras marítimas, las cuales ya habían ingresado con una carrera profesional, después, con el ingreso de las mujeres de línea en el año 1987, se forjaron en su carrera profesional dentro de la institución, en la cual, hasta la fecha, se han podido graduar no solo como administradoras marítimas, sino también han podido estudiar las diferentes carreras que la Escuela Naval ofrece, como ingeniería naval, o las carreras de superficie como: oceanografía, electrónica y administración.

Con el transcurrir del tiempo la mujer ha demostrado habilidades que le han dado un impulso notable al desarrollo de las relaciones de género, las cuales hace algunos años no pasaban de ser menospreciadas por su calidad de mujer. En este sentido, podemos mencionar que la historia de 
Colombia registra el heroísmo de muchas mujeres cuyo papel fue fundamental tanto en la lucha por la Independencia, como en la construcción de la nación. Muchas de ellas colaboraron con el Ejército Libertador como portadoras de correo, espías y divulgadoras de las ideas libertarias. (Forero, 2010) Sin embargo, el gran papel de las mujeres en nuestra independencia, solo en los primeros años de la República, Colombia fue uno de los últimos países del continente americano en concederles derechos políticos; y a partir de 1954 se le otorgó el derecho a la ciudadanía, cuando el entonces presidente de facto, el General Gustavo Rojas Pinilla, impuso una reforma constitucional que les dio el derecho a elegir y ser elegidas.

En la Armada Nacional de Colombia la mujer ingresa en 1984, inicialmente como profesionales, ya que la Escuela no poseía lugar para las féminas y siendo profesionales solo deberían recibir una breve instrucción y así su paso por la escuela sería muy corto.

Según (Davis, 2011) a partir del 2000 se ha multiplicado el ingreso de mujeres a las Fuerzas Militares de Colombia. Según cifras de las mismas Fuerzas Militares, en el 2015 el Ejército Nacional incorporó un $16 \%$ de mujeres a esta fuerza; la Armada Nacional, un 49,7\%, y la Fuerza Aérea, un $14 \%$. Estas estadísticas ratifican que en los últimos tiempos ellas han podido ocupar cargos diversos tanto de administración como de campo, comando de tropas e inteligencia. Gracias a estos cambios también han ejercido roles de poder, como es el caso de dos Brigadieres Generales del Ejército e incluso de la primera ministra de Defensa Nacional.

A mediados del siglo XX se ingresa a la mujer en los ejércitos ya que había mucha necesidad de mano de obra, al principio solo se les designaban tareas auxiliares y aquellas relacionadas con la medicina, pero con el tiempo las grandes capacidades de la mujer se fueron viendo reflejadas en sus acciones y así se les fue abriendo paso para desarrollar actividades diferentes a las anteriormente mencionadas.

El siglo XXI inicia para la Armada con un hecho muy relevante: porque en el 2000 se da el ingreso de las primeras mujeres oficiales de línea. Actualmente, las mujeres en la reserva de la Armada Nacional apoyan a las diferentes Fuerzas a cumplir su labor institucional, también cabe destacar que soportan las labores en las áreas administrativas, logística y de acción integral coordinada. De manera especial, en la Armada se han corroborado los esfuerzos de las mujeres por su profesionalización y por su ejercicio en especialidades que permiten el desarrollo de la propia institución en general, así como asegurar la protección de la soberanía del país. (Caicedo Angela, 2017)

Cabe destacar el realce en la parte académica. Estudiar las relaciones de género en un ámbito construido por y para hombres, de corte tradicional y patriarcal, basándonos en los estudios realizados al ambiente de trabajo que vive una mujer en un buque, es importante. Los estereotipos que dominan a la cultura occidental en lo que concierne a lo naval militar, definen al hombre como el que inicia la guerra y la mujer como hacedora de paz. Estos estereotipos van cambiando a medida que la mujer logra ganar espacios tradicionalmente masculinos. La incorporación de la mujer en las Fuerzas Armadas en especial en la Armada Nacional supone cambios trascendentales para la organización militar y para la sociedad. (Sanguinei Almeida, 2015)

Para 1997 ingresan las mujeres de línea, es decir que estudiarían su carrera profesional dentro de la institución, lo que indica que su duración dentro de la Escuela Naval tendría un periodo de cuatro años, lo cual generó muchos cambios para la marina, pues debió modificar no solo su infraestructura en todas las unidades, sino también debió empezar a generar un pensamiento de aceptación para poder crear una cultura militar que aceptara que la mujer hace parte de sus filas. La convivencia entre ambos géneros es un tema que puede causar intriga, pero lo más inquietante es reconocer cómo ha sido el trato entre mujeres y hombres dentro de un ambiente hostil, que desde que surgió fue diseñado para que solo hombres estuvieran en él, aun así, la mujer, gracias a todas sus capacidades, ha demostrado que sin importar cualquier tipo de ambiente puede desempeñar el cargo que se le imponga.

Ingresar a la mujer en los ejércitos internacionales refleja un modelo social, además ellas contribuyen a que el desarrollo de las fuerzas sea mejor. En Europa la mujer puede ocupar casi cualquier empleo existente, y el ámbito militar no es la excepción, pues estas han ocupado puestos importantes, por ejemplo: en países como España y Noruega no tienen ninguna restricción para la 
incorporación de la mujer en puestos y empleos, incluyendo su participación en tripulaciones de submarinos, a diferencia de otros países con armadas que también están tripuladas por mujeres, dándole así una relevancia al género femenino, demostrando que ser mujer no significa más que un género. (INZA, 2015).

El proceso de formación de una mujer profesional y una de línea son muy diferentes, la que viene con una carrera terminada pasa por la Escuela con el fin de aprender los programas militares y navales, además según su profesión su duración va de seis meses a un año máximo, a diferencia de aquellas mujeres que ingresan siendo bachilleres, las cuales deben iniciar su pregrado en la institución, el cual tiene un tiempo de duración de cuatro años, en los cuales deben cumplir con todos los requerimientos exigidos por la Escuela Naval, como son la doctrina militar, los programas académicos, los cursos navales, las exigencias físicas y demás, pero principalmente deben acostumbrarse a vivir en un ambiente que desde sus orígenes no fue creado para ellas y que con el tiempo se ha venido adaptando, debido a que hombres y mujeres en la Escuela Naval cumplen con las mismas exigencias, el género no distingue quien debe hacer las cosas, ya que todos son vistos desde la misma perspectiva, como futuros Oficiales Navales independientemente de si son hombres o mujeres.

Con el ingreso de las primeras féminas de línea a la Escuela Naval en 1997, lo que se quería comprobar era si las colombianas resistirían toda la carrera naval, y aunque su ingreso parecía fácil era todo un reto poder asumir esta posición, pues con el ingreso de mujeres administrativas no era tan notorio el cambio, ya que su paso por la institución era muy corto, pero con el de las mujeres de línea se podrían evidenciar todos los cambios, y hasta la fecha las colombianas han demostrado que para ellas asumir todos estos retos no fue más que un desafío superado.

Entre las consecuencias que esto ha generado podemos mencionar: el desconocimiento de la cultura naval y realce al género que por mucho tiempo en la antigüedad fue menospreciado, pero no tomaban en cuentan las importantes cualidades que la mujer tiene para el desarrollo de la visión de nuestra Alma Mater. También se evidencia que en sus inicios la cadete de línea solo podía pertenecer a una sola especialidad, la cual era el cuerpo logístico. En donde se graduarían como administradoras marítimas especializadas en logística y tendrían la oportunidad de continuar con la carrera naval hasta alcanzar el grado de almirante.

Cabe destacar que en 1976 el país permitió la incorporación de la mujer en las filas castrenses, como oficiales administrativas del Ejército de Colombia en la Escuela de Cadetes General José María Córdoba. Al principio solo se les designaban tareas auxiliares, administrativas y aquellas relacionadas con la medicina, pero con el tiempo las grandes capacidades de la mujer se fueron viendo reflejadas en sus acciones y así se les fue abriendo paso para desarrollar actividades diferentes.

En la Armada Nacional de Colombia las mujeres ingresan en 1984. Al principio solo como profesionales, ya que la Escuela no poseía lugar para ellas, y siendo profesionales solo deberían recibir una breve instrucción y así su paso por la escuela sería muy corto. En 1997 se da el ingreso de las primeras mujeres oficiales de línea, es decir que su preparación sería dentro de la Escuela Naval de Cadetes "Almirante Padilla" por un periodo de cuatro años. Actualmente, las mujeres de la Armada Nacional de Colombia apoyan a las diferentes Fuerzas a cumplir su labor institucional, también cabe destacar que soportan las labores en las áreas administrativas, logística y de acción integral coordinada. De manera especial en la Armada se han corroborado los esfuerzos de las mujeres por su profesionalización $\mathrm{y}$ por $\mathrm{su}$ ejercicio en especialidades que permiten el desarrollo de la institución, así como asegurar la protección de la soberanía del país. (Caicedo Angela, 2017).

De acuerdo a la entrevista realizada a la señora CN(RA) Cecilia María Páez Flórez, se deduce que desde sus inicios la mujer en la Escuela Naval se visualizó como el sexo débil de la sociedad, el trato hacía ellas era mínimo y sobre todo delicado, pero en realidad la mujer es visualizada desde el punto de vista profesional como alguien que sin problema puede ocupar cualquier cargo que requiera la Armada de Colombia, pues, si bien es cierto, todo fue creado para ser ocupado por hombres, pero la destreza de las mujeres ha demostrado que esos cargos fácilmente y de manera muy efectiva se desarrollan por ellas, demostrando que pueden 
cumplir con todos los estándares necesarios para ser una oficial naval.

CN(RA) Cecilia María Páez Flórez afirma que cuando la mujer ingresa a la Escuela Naval por primera vez en 1984 solo fue aceptada para el cuerpo administrativo, es decir, mujeres que ya habían realizado una carrera profesional. Desde ese momento iniciaron los cambios, como, por ejemplo: los laborales, en el tema de convivencia, el trato con el personal. Su formación era de periodos cortos y hasta ahí no era tan reflejada su influencia, el verdadero cambio surgió cuando en 1997 ingresan las primeras féminas de línea, pues no solo se manifestó en el hecho de convivir cuatro años con personas de otro género, realizando las mismas actividades, sino que después se plasma de manera significativa en el ámbito operacional. Ingresar a la mujer a las filas de la Armada de Colombia ha brindado mucha evolución a la misma, pues, la Armada es como una empresa cualquiera del país, en la cual no hay exclusión, por lo tanto, el ingreso de la mujer se refleja como una oportunidad de desarrollo profesional en los diferentes ámbitos que desempeñe. La Armada de Colombia al contar con el punto de vista de la mujer ha podido desarrollar actividades diferentes a las que venía realizando habitualmente. La institución estuvo retardada a comparación de las otras marinas del mundo para ingresar a la mujer en sus filas, es cierto que esto requeriría una inversión, pues debió ser modificada toda su estructura en las unidades y el cambió de pensamiento fue algo que costó mucho trabajo, ya que no es fácil modificar los ideales de una cultura de un día para otro, pero igual es algo que debía suceder, todo debe ir cambiando y mejorando. Nunca se pondría en duda el ingreso de la mujer a la Armada de Colombia, porque ella está capacitada para desempañar cualquier cargo, estudiar cualquier carrera y destacarse a bordo de las unidades sin ningún problema, además, la institución es como cualquier otra de educación superior que ofrece un pregrado, solo que llevan un régimen de vida diferente, lo que hace que las personas que allí ingresan sean vistas desde otra perspectiva.

El trato en la Escuela Naval entre hombres y mujeres es igual en cualquier ambiente, no existen preferencias, pues realizan las mismas actividades, como trotes, mover materiales y demás, y esto hace que la relación entre estos dos géneros sea buena ya que crean lazos de amistad, conviven en un ámbito académico, comparten la misma aula y gustos; y se nota cómo el hombre no rechaza o discrimina a la mujer y ellas por tal razón no los consideran machistas. Aun así, para que esta relación sea más estrecha la solución sería que no existieran tantas prohibiciones, pues el cohibir a las personas de algo las incita a que lo hagan más, lo convierte en algo más llamativo, afirma CN(RA) Cecilia María Páez Flórez.

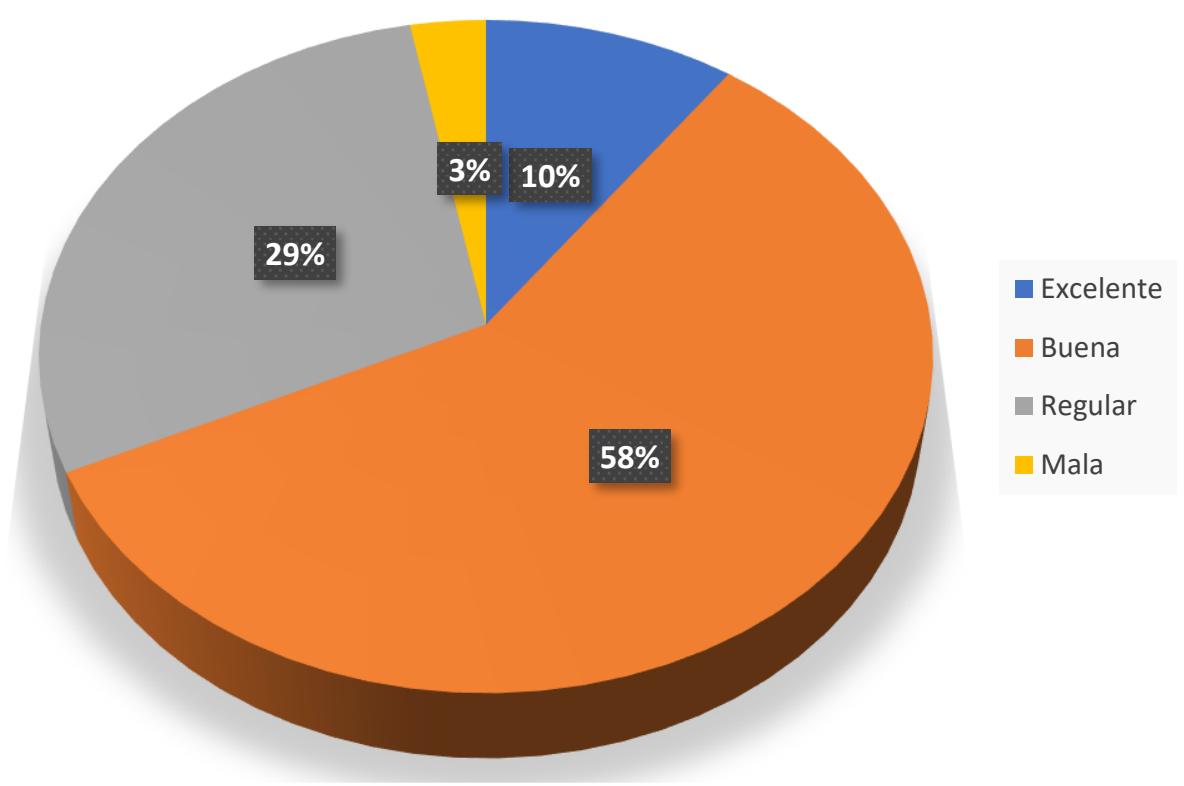

Figura 1. Relaciones entre hombres y mujeres.

Fuente: Los autores. 
Ella también dice que para la sociedad el ingreso de la mujer a las filas de las Fuerzas Armadas como lo es la Marina causó un impacto un poco despectivo, pues la gente cree que por ser militar se pierde la feminidad, cuando de ninguna manera una mujer por utilizar botas, pantalones y demás accesorios militares pierde su esencia. El hecho de hablar fuerte, ser un poco más seria, tener el cabello recogido, y realizar actividades que en la vida cotidiana generalmente son resueltas por hombres no debe hacer que la mujer sea vista de otra manera, más que como una persona que simplemente cumple estándares diferentes a los de la zona de confort, pero que mantiene su esencia.

También se indagó cuáles son las personas que día a día viven las diferentes situaciones entre hombres y mujeres que pueden presentarse en ciertos casos y se concluyó lo siguiente:

Al realizar una encuesta a los cadetes del batallón de la Escuela Naval se evidenció que el $58 \%$ del personal afirmó que la relación con personas de otro género es buena, ya que existe respeto, los hombres consideran que la mujer es un excelente complemento en la realización de cualquier tipo de actividad, ya que su apoyo y su punto de vista es crítico y de mucho valor. Ellos afirman que las mujeres tienen un ejercicio de mando que en ocasiones es mejor que el suyo, lo que hace que sus relaciones sean buenas porque les ayudan a manejar diferentes situaciones en cualquier contexto. Ambos géneros están de acuerdo con que la relación es buena por la disciplina impartida en la institución, lo que logra que los dos acepten el valor del otro.
El 29\% dice que la relación es regular, pues la mayoría coincide en que el problema es que existan relaciones interpersonales y esto provoca que de algún modo se cree alguna preferencia o que la personal tienda a confundir las cosas personales con las profesionales, el $10 \%$ afirma que la relación es excelente ya que no tienen problema con alguien de otro género, y el 3\% manifiesta que sí existe una mala relación entre hombres y mujeres por la falta de tolerancia.

La encuesta también lleva a concluir cómo es el trato entre ambos géneros, indicando que el $74 \%$ de los alumnos consideran que llevan un buen trato con alguien de otro género, ya que existe respeto y este siempre debe ser mutuo, también porque hay aceptación del mismo, sin embargo, el 13\% asegura que la relación es regular, pues algunos hombres aún tienen un pensamiento machista así que eso conlleva a que la relación no sea tan efectiva, y que algunas veces se den ambientes de conflicto por la diferencia de pensamiento del otro. El $8 \%$ avala que la relación es excelente porque todos conocen el valor del respeto y que tratan a las demás personas como desean ser tratados, a pesar de todo el $5 \%$ indica que si existe una mala relación porque así como hay personas que tratan a las demás con respeto, hay otras que no lo hacen, y como hay algunas que aún tienen un pensamiento machista esto genera que ciertos tratos sean vistos como maltrato, también porque algunas veces hay abusos de confianza o rivalidad de unos con otros simplemente por ser hombres o mujeres, ya que en ocasiones ellas, por demostrar fortaleza, los ven como su competencia directa.

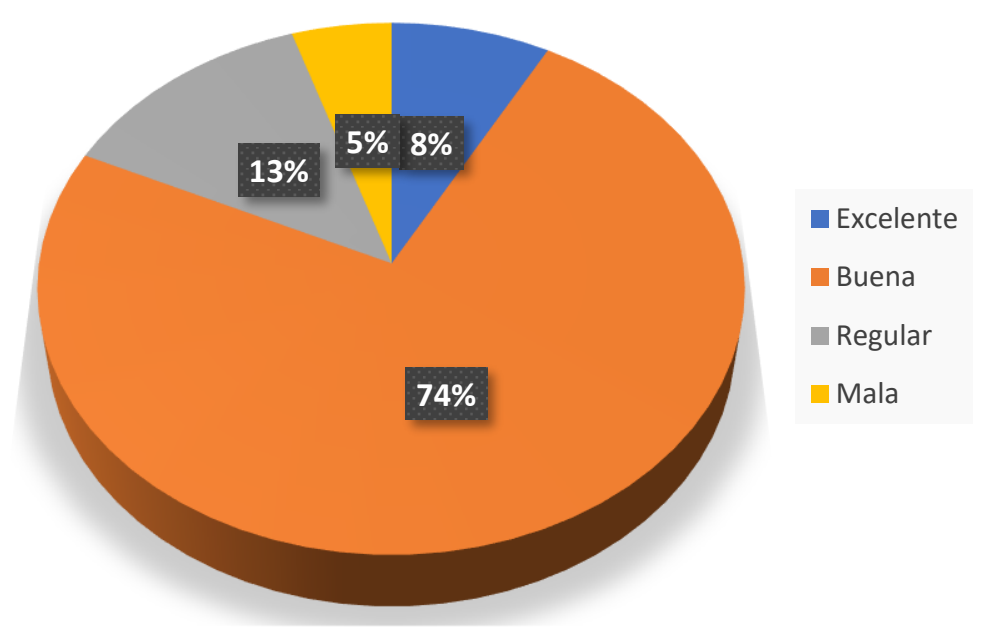

Figura 2. Trato entre hombres y mujeres.

Fuente: Los autores. 


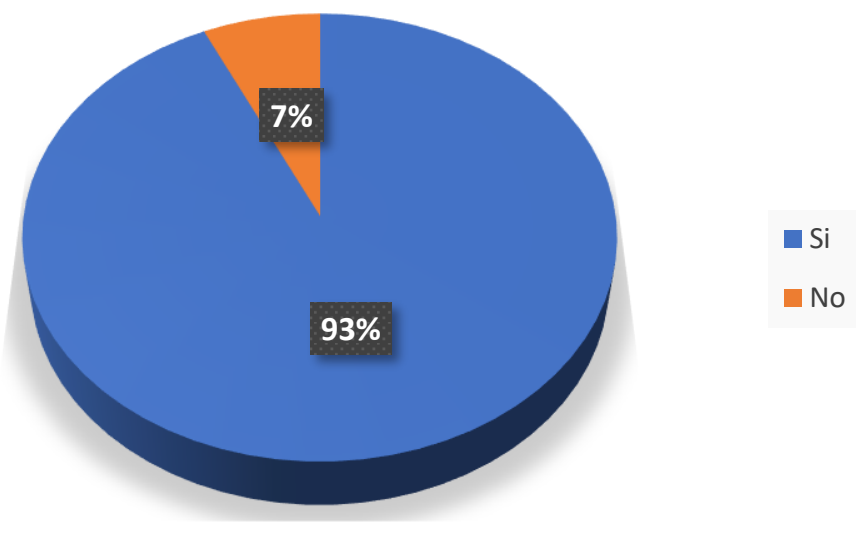

Figura 3. Visualiza a la mujer dirigiendo a la Escuela Naval.

Fuente: Los autores.

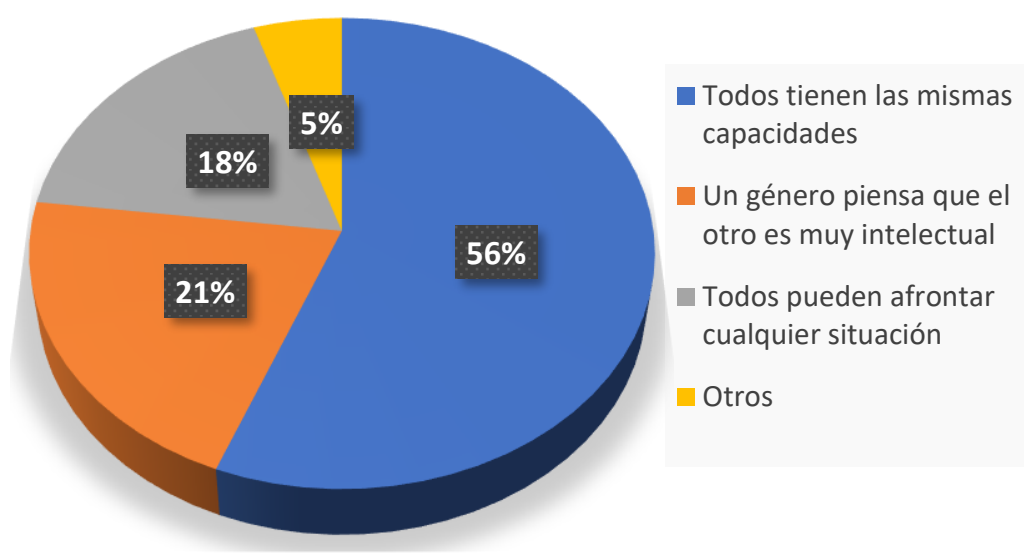

Figura 4. Capacidades.

Fuente: Los autores.

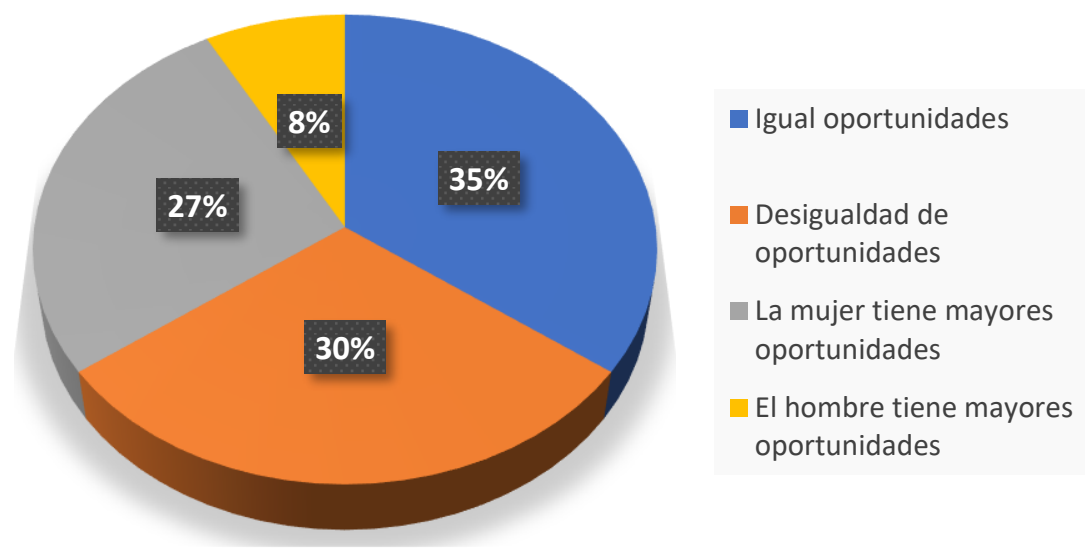

Figura 5. Oportunidades.

Fuente: Los autores.

Aunque la mujer ingresó a la Armada hace unos 36 años, aún no hay ninguna que alcance la antigüedad necesaria para ocupar dicho cargo, pero, teniendo en cuenta que ese momento llegará pronto, se trató de conocer si la comunidad estudiantil está preparada o visualiza la institución direccionada por una mujer, a lo que el $93 \%$ afirma que, si visualiza a la Escuela Naval al mando de una fémina, el otro $7 \%$ manifiesta que realmente no está preparado para que la escuela sea direccionada por una mujer. Con esto se puede ratificar cómo realmente existe el machismo en la institución, aunque sea en minoría, 
pues el hecho de no visualizar a la Escuela direccionada por una mujer es pensar que esta no podrá desempeñar bien su cargo, tal vez por pensamientos así, es que el trato de unos pocos a otros no es totalmente bueno.

También se requirió analizar cómo un género ve al otro por sus capacidades, en lo que el 56\% afirmó que tanto hombres como mujeres tienen las mismas capacidades físicas para desarrollar cualquier tipo de ejercicio o actividad, el $21 \%$ concluyó que ve al género contrario como capaz de desarrollar cualquier actividad intelectual, ya que considera que hombres y mujeres son igual de inteligentes, de lo contario no estarían dentro de la institución. El 18\% dice que todos están preparados para afrontar cualquier tipo de situación que amerite de sus capacidades, y el 5\% restante concluye consideraciones diferentes, como que a algunos les falta espíritu de cuerpo, que considera al otro género como valiente, que hay unos con mejor mando que otros o que hay mucho potencial.

De igual forma se verificó cómo consideran al otro género, pero según sus oportunidades, en lo que el 35\% coincidió en que todos tienen las mismas oportunidades. El $30 \%$ afirma que hay desigualdad de oportunidades para ambos, el $27 \%$ dice que las mujeres tienen más oportunidades que los hombres y el $8 \%$ restante coincide con que los hombres tienen mayor oportunidad que las mujeres

Se les preguntó a los alumnos ¿Qué harían para mejorar la relación con el otro género? y estas fueron algunas de sus respuestas:

- Buscar mayor igualdad de oportunidades para ambos géneros.

- Que haya charlas donde se resalte la importancia tanto de hombres y mujeres en la realización de cualquier tipo de actividad.

- Que las sanciones y felicitaciones sean iguales para ambos.

- Fomentar más el trabajo en equipo en donde un género note como necesita del otro para cumplir ciertos objetivos.

- Buscar la manera de que exista más comunicación.
- Que la mujer sea tenida más en cuenta en actividades importantes, como comisiones y demás.

- Que haya integraciones para que deje de existir machismo y discriminación.

- Confirmar con actividades que la mujer no es el 'sexo débil'.

En otro aparte, al indagar y analizar las herramientas disponibles en la institución para la integración entre ambos géneros, se llegó a la deducción de que, aunque hombres y mujeres se relacionen todo el tiempo, no existen estrategias claras que inciten a que se respeten y valoren, más que por su condición de género, por la importancia que tiene el uno para el otro y lo complementarios que pueden ser.

Estás herramientas podrían ser aquellas que permitan valorizar al otro género, por ejemplo: charlas en donde sean los hombres quienes desde su punto de vista digan porqué consideran que la mujer es importante, otra opción podría ser mediante didácticas que lleven a necesitar hombres de mujeres y viceversa, para la realización de un objetivo.

Las herramientas para integrar ambos géneros pueden ser muchas y de diferentes maneras, porque para que la convivencia sea mejor no solo basta con compartir diariamente, si no buscar entender los problemas que según el género se puedan presentar.

\section{Conclusiones}

La Armada Nacional de Colombia es una institución que decidió integrar a la mujer en el año 1984, lo cual hizo que surgieran diferentes cambios tanto estructurales como de pensamiento. La mujer desde su ingreso ha demostrado que no importa el cargo que le sea impuesto, siempre lo ha desarrollado. Para la Escuela Naval, al ser la Alma Mater de la oficialidad Naval, es muy importante tener en cuenta cómo es la relación entre hombres y mujeres en el desarrollo de cualquier actividad, pues como su lema lo indica "aquí nace la Marina" desde sus aulas, alojamientos, calles y tradiciones se forma al futuro oficial, el cual, según todas sus enseñanzas, se desempeña en las unidades. Es importante que exista una relación estrecha entre ambos géneros, no 
solo por ser compañeros, si no por el hecho de valorar sus desempeños. Según las encuestas realizadas, las relaciones de género no son malas en su mayoría, se cree que tal vez hay un déficit en las oportunidades que puede tener el uno u el otro por ser hombre o mujer, pero en general consideran que poseen las mismas capacidades y que de una u otra manera son complementarios, ya sea en el ejercicio de mando o en el desarrollo de actividades específicas.

La Escuela Naval nunca ha sido direccionada por una mujer, pero en su gran mayoría los alumnos están dispuestos y enfocados al cambio, a experimentar como sería este direccionamiento. A pesar de que existe un pensamiento machista inferior, el cual no puede visualizar la institución de esta manera, el resto de los alumnos si logra comprender lo impactante e importante que sería si se llevara a cabo.

Aunque en sus inicios la mujer era tratada remotamente y de manera delicada, hoy en día el trato hacia ellas no tiene diferencia con el de los hombres, pues la mujer no es excluida de realizar las actividades propuestas por el batallón; por el contrario, busca integrarla en todas las tareas, ya que es importante su participación.

Se nota cómo todos los cadetes tienen clara la importancia de respetarse los unos a los otros como valor fundamental, pues nadie desea ser tratado de mala manera, por tal motivo tanto hombres como mujeres se tratan de manera cordial con el fin de recibir lo mismo, además, el hombre busca hacer que ese mínimo de machismo no afecte sus relaciones profesionales y académicas.

\section{Trabajos futuros}

Se recomiendan para futuros trabajos de investigación sobre la caracterización de las relaciones de género en la Escuela Naval de cadetes "Almirante Padilla", indagar no solo con el personal de cadetes del batallón, sino con los alumnos en general y oficiales de la Escuela Naval, para poder tener una referencia de cómo se ven las relaciones entre ambos géneros. También es recomendable entrevistar a una gran mayoría de oficiales que tuvieron la experiencia desde el ingreso de las primeras féminas de línea y su paso por la Escuela Naval, para evidenciar con más argumentos la evolución que ha tenido la Institución. También se aconseja el apoyarse en herramientas de investigación como encuestas y entrevistas; realizar talleres que demuestren cómo es el trato hacia el otro género y cómo se visualizan los unos a los otros mediante ejercicios reales, para así proponer estrategias que permitan realizar proyectos en los que se pueda interactuar de manera práctica para la recolección de información.

\section{Referencias}

Caicedo Ángela María, M. L. (2017). mujeres militares. Obtenido de http://www2.unwomen.org

Davis, K. (2011). Sex, gender and cultural intelligence.

Forero. (2010). participacion de la mujer en la independencia, revista fuerzas armadas.

INZA, B. P. (22 de 10 de 2015). Estudios de politica exterior. Obtenido de https://www.politicaexterior.com/articulos/econ omia-exterior/participacion-de-la-mujer-en-lasfuerzas-armadas/

Sangueti Almeida. (2015). ALMEIDA. Obtenido de https://www.colibri.udelar.edu.uy/jspui/bitstrea m/20.500.12008/4991/1/TCP_AlmeidaSanguine tNadia.pdf 